\title{
Evolving Neural Networks for Chlorophyll-a Prediction
}

\author{
Xin Yao \\ School of Computer Science \\ The University of Birmingham \\ Edgbaston, Birmingham B15 2TT \\ U.K. \\ Email: x.yao@cs.bham.ac.uk
}

\author{
Yong Liu \\ The University of Aizu \\ Tsuruga, Ikki-machi, Aizu-Wakamatsu \\ Fukushima 965-8580 \\ Japan \\ Email: yliu@u-aizu.ac.jp
}

\begin{abstract}
This paper studies the application of evolutionary artificial neural networks to chlorophyll-a prediction in Lake Kasumigaura. Unlike previous applications of artificial neural networks in this field, the architecture of the artificial neural network is evolved automatically rather than designed manually. The evolutionary system is able to find a near optimal architecture of the artificial neural network for the prediction task. Our experimental results have shown that evolved artificial neural networks are very compact and generalise well. The evolutionary system is able to explore a large space of possible artificial neural networks and discover novel artificial neural networks for solving a problem.
\end{abstract}

\section{Introduction}

Chlorophyll-a prediction is a highly nonlinear problem. Previous attempts using artificial neural networks (ANNs) have achieved only limited success [1, 2]. According to Recknagel [2], the background of the problem can be described as follows:

Lake Kasumigaura is situated in the South-Eastern part of Japan. It has a large and shallow water body where no thermal stratification occurs. The annual water temperature of the lake ranges from $4^{\circ} \mathrm{C}$ to $30^{\circ} \mathrm{C}$ in summer. Due to high external and internal nutrient loadings, the primary productivity of the lake is extremely high, ..., and favours harmful blue-green algae such as Microcystis spp, Oscillatoria and Anabaena flos aquae. As algal succession changes species abundance year by year, it is very difficult to causally determine and predict algal blooms in Lake Kasumigaura.

Accurate prediction of chlorophyll-a and other blue-green algae will no doubt be very useful in protecting the fresh-water environment.

Recknagel $[1,2]$ has recently proposed to use feed-forward ANNs to predict and model species abundance and succession of blue-green algae in Lake Kasumigaura. A strictly three-layer feedforward ANN (without any shortcut connections between input and output layer) was used. The ANN has 10 input nodes and one output node. The 10 inputs nodes include water temperature, solar radiation, secchi depth, rotifera density, cladocera density, etc. The single output indicates the abundance of chlorophyll-a. All inputs and output were real-valued numbers. The number of hidden nodes in the hidden layer was tuned manually and then fixed during ANN training. The experimental results reported by Recknagel $[1,2]$ were very promising, although more improvements can be made.

This paper proposes to use evolution to design ANN architectures automatically. The evolutionary approach to ANN architecture design can relieve human beings from the tedious trial-and-error 
architecture tuning task (e.g., adjusting the number of hidden nodes). It is also capable of exploring a much larger space of possible ANNs and finding the most suitable ANN for chlorophyll-a prediction in Lake Kasumigaura.

The remaining part of this paper will be organised as follows: Section 2 introduces an evolutionary system for automatica design of ANNs. Section 3 describes the experiments we carried out for chlorophyll-a prediction. Section 4 concludes the paper.

\section{Evolving Artificial Neural Networks}

Most of the studies in ANNs have concentrated on the training issue, i.e., updating connection weights of an ANN in order to minimise an error function [3]. The ANN architecture is often designed manually through a tedious trial-and-error process and assumed to be fixed during training. This approach often leads to unsatisfactory ANNs since manually exploring a large number of different ANN architectures is impractical. The success of ANN applications relies heavily on prior knowledge an ANN designer has about the application as well as ANN theories.

Finding a near optimal ANN for an application task is a very difficult task. Some heuristic algorithms have been proposed to construct or prune ANNs automatically [4, 5]. However, such heuristic methods may be trapped in a poor local optimum in the ANN architecture space due to their hill-climbing nature. Evolutionary algorithms (EAs) are a class of population-based stochastic search algorithms [6]. They are very good at global search in a multimodal and complex space where the search surface (i.e., landscape) may not be continuous. They can be used to find a near optimal ANN automatically without human intervention.

EPNet [7] is an automatic system that evolves ANN architectures and connection weights simultaneously. It has been applied to many benchmark problems with success. EPNet uses an evolutionary programming (EP) algorithm $[8,9]$ to evolve ANN architectures. It uses only selection and mutations. The selection used is a nonlinear ranking scheme [10]. The five mutations used are hybrid training, (hidden) node deletion, connection deletion, connection addition, and (hidden) node addition. These five mutations are attempted sequentially in the aforementioned order in order to encourage the parsimony of ANNs. In any given generation, only one of the mutations will be used successfully. A mutation is regarded as unsuccessful when the offspring does not improve the performance of the parent to certain degree (i.e., over certain threshold).

Hybrid training is the only mutation that updates the connection weights of an ANN. The other four are all architecture modification mutations. The hybrid training consists of a back-propagation (BP) algorithm with adaptive learning rates and a simplified simulated annealing (SA) algorithm. The SA algorithm is used to help BP escape from poor local minima. An important feature about our architectural mutations is that there is always a partial training process after a successful mutation. The motivation behind such an additional training process is to bridge the behavioural (functional) gap between a parent and its offspring caused by the architectural mutation. The training is partial because only a fixed number of epochs are allowed.

A connection is added to or deleted from an ANN probabilistically depending on how important the connection is in the ANN [7]. A hidden node is deleted at random, but a hidden node is added by splitting an existing node in order to better maintain the behavioural link between the parent and its offspring $[7,11]$.

EPNet is capable of evolving general feed-forward ANNs with shortcuts. Both the number of hidden nodes and connectivity among nodes are subject to evolution. Both ANN architectures and connection weights will be determined by EPNet at the end of evolution. The next section presents some experimental results of applying EPNet to the chlorophyll-a prediction problem. 


\section{Evolving Artificial Neural Networks for Chlorophyll-a Predic- tion}

In order to compare our results with previous work, we have followed as closely as possible previous experimental setup described in [2]. The limnological time series for 10 years between 1984 and 1993, inclusively, were used in our experiments. The experiment was divided into two parts. The first part used the data in 1984 and 1985 as the training data to evolve the first ANN. Then the ANN was tested on the 1986 data. The 1986 data was not used in evolving ANNs. The second part of the experiment used the data between 1987 and 1992 as the training data to evolve the second ANN. Then the ANN was tested on the 1993 data. Similarly, the 1993 data was not used anywhere in evolving ANNs. Using the 1986 and 1993 data as the independent testing data was suggested by Recknagel $[1,2]$ because they represent typical years for blooms of Microcystis and Oscillatoria, respectively.

As a preprocessing step, the original data were rescaled linearly to between 0.1 and 0.9 . The input to an ANN consists current input conditions and output conditions in the past seven days. More details about these data were given in $[1,2]$.

The normalised root-mean-square (RMS) error $E$ was used to evaluate the performance of EPNet, which is determined by the following formula [12]:

$$
E=\frac{\left\langle\left[x_{p r e d}(t, \Delta t)-x(t+\Delta t)\right]^{2}\right\rangle^{\frac{1}{2}}}{\left\langle(x-\langle x\rangle)^{2}\right\rangle^{\frac{1}{2}}}
$$

where $\Delta t$ indicate the prediction time span (e.g., $\Delta t=1$ implies predicting the value of the next time step), $x_{\text {pred }}(t, \Delta t)$ is the prediction of $x(t+\Delta t)$ based on the current state $x(t)$, and $\langle x\rangle$ represents the expectation of $x$. As pointed out by Farmer and Sidorowich[12], "If $E=0$, the predictions are perfect; $E=1$ indicates that the performance is no better than a constant predictor $x_{\text {pred }}(t, \Delta t)=\langle x\rangle . "$

In order to obtain statistically significant results, we have carried out 30 independent runs from different random initial conditions for each experiment. Table 1 summarises the average result from EPNet over 30 runs for the first part of the experiment when the 1984 and 1985 data were used for training (i.e., evolution) and the 1986 data were used for independent testing. Figure 1 shows the best prediction produced by EPNet on the training and testing data, respectively.

Table 2 summarises the average result from EPNet over 30 runs for the second part of the experiment when the 1987 to 1992 data were used for training (i.e., evolution) and the 1993 data were used for independent testing. Figure 2 shows the best prediction produced by EPNet on the training and testing data, respectively.

Table 1. The average results produced by EPNet over 30 independent runs for the chlorophyll-a prediction problem in Lake Kasumigaura from 1984 to 1986.

\begin{tabular}{|l|c|c|c|c|}
\hline & Mean & Std Dev & Min & Max \\
\hline \hline Number of Connections & 57.70 & 23.36 & 24 & 135 \\
\hline Number of Hidden Nodes & 2.83 & 1.37 & 1 & 7 \\
\hline Error on Training Data (1984-1985) & 0.0128 & 0.0014 & 0.0099 & 0.0161 \\
\hline Error on Testing Data (1986) & 0.0868 & 0.0077 & 0.0601 & 0.1021 \\
\hline
\end{tabular}

It is clear from Figures 1 and 2 that both evolved ANNs generalise very well to the independent testing data. Almost all the changes in the time series have been captured by the ANNs. In terms of ANN architectures, Tables 1 and 2 give the average number of hidden nodes and connections in the evolved ANNs. The average number of hidden nodes used in an ANN for both experiments is less than three. The average number of connections is under 58 and 44, respectively, for the two 


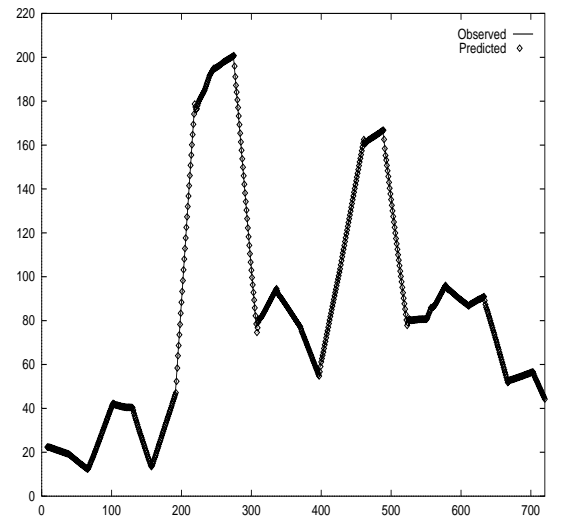

(a)

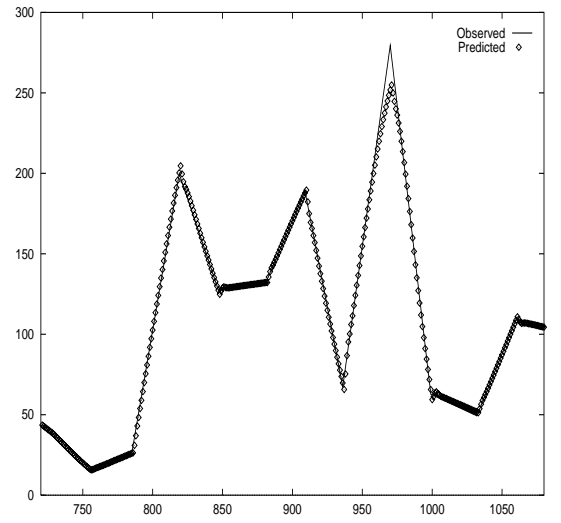

(b)

Figure 1. (a) The performance of the best ANN evolved by EPNet for chlorophyll-a prediction in Lake Kasumigaura for 1984 to 1985. (b) The performance of the best ANN evolved by EPNet for chlorophyll-a prediction in Lake Kasumigaura for 1986. The time span is $\Delta t=1$.

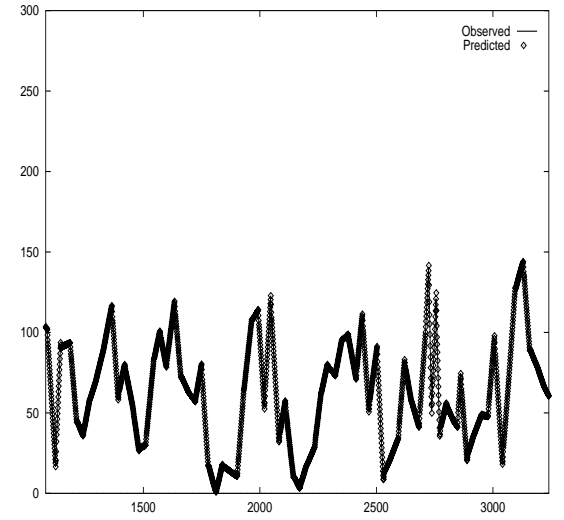

(a)

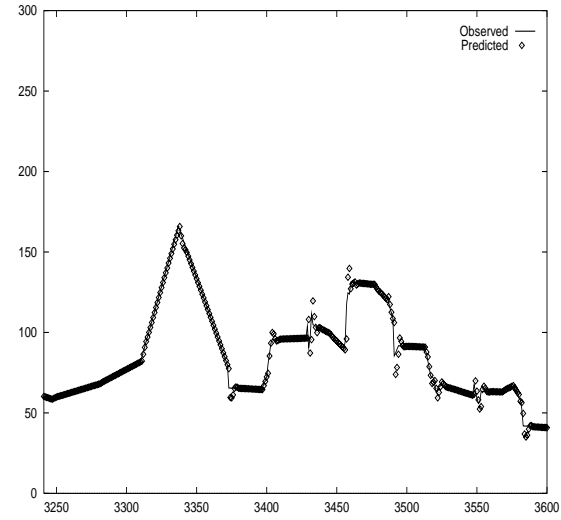

(b)

Figure 2. (a) The performance of the best ANN evolved by EPNet for chlorophyll-a prediction in Lake Kasumigaura for 1987 to 1992. (b) The performance of the best ANN evolved by EPNet for chlorophyll-a prediction in Lake Kasumigaura for 1993. The time span is $\Delta t=1$.

Table 2. The average results produced by EPNet over 30 independent runs for the chlorophyll-a prediction problem in Lake Kasumigaura from 1987 to 1993.

\begin{tabular}{|l|c|c|c|c|}
\hline & Mean & Std Dev & Min & Max \\
\hline \hline Number of Connections & 43.80 & 16.28 & 22 & 83 \\
\hline Number of Hidden Nodes & 2.47 & 0.86 & 1 & 5 \\
\hline Error on Training Data (1987-1992) & 0.0200 & 0.0010 & 0.0182 & 0.0224 \\
\hline Error on Testing Data (1993) & 0.1197 & 0.0061 & 0.1107 & 0.1396 \\
\hline
\end{tabular}


experiments. These results illustrate that evolution can automatically generate compact ANNs that generalise well for the chlorophyll-a prediction problem.

\section{Conclusions}

Accurate prediction of chlorophyll-a and other blue-green algae in fresh water lakes can provide ecologists and environmentalists with valuable information for controlling major outbreaks of these algae and protecting the environment. This paper has proposed to use evolution to automatically design ANNs for chlorophyll-a prediction. Evolution provides a powerful technique for exploring a large space of all possible ANNs. It can relieve human beings from designing ANNs manually through trial-and-error. It can also discover some novel ANNs that can hardly hit upon by human designers through manual search. The smaller-than-expected ANNs evolved by EPNet support this argument.

Although the experiments presented in this paper deal only with the chlorophyll-a prediction problem in Lake Kasumigaura, the approach and techniques introduced in this paper can be applied to other problems. We expect to obtain similarly good results for other data with different blue-green algae in different fresh-water lakes. This will be our future work.

\section{References}

[1] F. Recknagel, "ANNA - artificial neural network model for predicting species abundance and succession of blue-green algae," Hydrobiologia, vol. 349, pp. 47-57, 1997.

[2] F. Recknagel, T. Fukushima, T. Hanazato, N. Takamura, and H. Wilson, "Modelling and prediction of phyto- and zooplankton dynamics in lake kasumigaura by artificial neural networks," Lakes and Reservoirs, vol. in press, 1998.

[3] D. R. Hush and B. G. Horne, "Progress in supervised neural networks," IEEE Signal Processing Magazine, vol. 10, no. 1, pp. 8-39, January 1993.

[4] S. E. Fahlman and C. Lebiere, "The cascade-correlation learning architecture," in Advances in Neural Information Processing Systems 2 (D. S. Touretzky, ed.), pp. 524-532, Morgan Kaufmann, San Mateo, CA, 1990.

[5] R. Reed, "Pruning algorithms - a survey," IEEE Trans. on Neural Networks, vol. 4, no. 5, pp. 740-747, 1993.

[6] D. B. Fogel, "An introduction to simulated evolutionary optimisation," IEEE Trans. on Neural Networks, vol. 5, no. 1, pp. 3-14, 1994.

[7] X. Yao and Y. Liu, "A new evolutionary system for evolving artificial neural networks," IEEE Transactions on Neural Networks, vol. 8, no. 3, pp. 694-713, 1997.

[8] L. J. Fogel, A. J. Owens, and M. J. Walsh, Artificial Intelligence Through Simulated Evolution. New York, NY: John Wiley \& Sons, 1966.

[9] D. B. Fogel, Evolutionary Computation: Towards a New Philosophy of Machine Intelligence. New York, NY: IEEE Press, 1995.

[10] X. Yao, "An empirical study of genetic operators in genetic algorithms," Microprocessing and Microprogramming, vol. 38, pp. 707-714, 1993.

[11] X. Yao, "The importance of maintaining behavioural link between parents and offspring," in Proc. of the 1997 IEEE Int'l Conf. on Evolutionary Computation (ICEC'97), Indianapolis, USA, pp. 629-633, IEEE Press, New York, NY, April 1997.

[12] J. D. Farmer and J. J. Sidorowich, "Predicting chaotic time series," Physical Review Letters, vol. 59, no. 8, pp. 845-847, 1987. 